\title{
Syndrome de Marfan et chirurgie implantaire
}

\section{Marfan syndrome and implant surgery}

\author{
SIHAM TAISSE ${ }^{1}$, MOUNIA EL BOUHAIRI ${ }^{1}$, FATIMA-ZAHRA EL OMARI ${ }^{2}$, IHSSANE BENYAHYA ${ }^{1}$
}

\section{RÉSUMÉ}

Le syndrome de Marfan (SM) est une affection rare, à transmission autosomique dominante. Les manifestations cliniques sont principalement cardiovasculaires et musculosquelettiques.

Un patient consulte au CCTD pour le remplacement de la 11 par une couronne sur implant. L'anamnèse révèle qu'il est atteint d'un SM. Un bilan global doit donc être réalisé et, pour informer correctement le patient, on doit répondre aux questions suivantes:

- Une intervention de chirurgie implantaire est-elle compatible avec l'état général du sujet?

- L'ostéointégration va-t-elle s'effectuer correctement?

- L'analyse de ces différents éléments permet-elle de répondre favorablement au patient ? (Med Buccale Chir Buccale 2008 ; 14: 227-231).

mots clés: syndrome de Marfan, cavité buccale, implant

médecine buccale chirurgie buccale

VOL. $14, \mathrm{~N}^{\circ} 4$ 2008

page 227

\section{SUMMARY}

Marfan syndrome is an autosomal dominant connective tissue disorder, which displays variable manifestations in the cardiovascular and skeletal systems.

A 17 year old male presented to the CCTD (oral consultation and treatment centre), to substitute the 11 with an implant. In front of his skeletal deformities, we have some questions to answer:

- Can the patient general state indicate an implant surgery?

- Is the osteointegration possible in this case?

- Through this clinic case, we will discus the possibility to response to this patient ask. (Med Buccale Chir Buccale 2008 ; 14: 227-231).

key words: Marfan syndrome, oral cavity, implant

1. Service d'Odontologie Chirurgicale Centre de consultation et de traitements dentaires (CCTD) Casablanca Maroc

2. Service de Pédodontie-Prévention Centre de consultation et de traitements dentaires (CCTD) Casablanca Maroc

Demande de tirés à part:

Siham Taisse Service d'odontologie chirurgicale Rue Abou Al Alaa Zahar 21100 B.P 9157 Mers Sultan Casablanca Maroc sihamtaisse@yahoo.fr 
médecine

buccale

chirurgie

buccale

VOL. $14, \mathrm{~N}^{\circ} 4$ 2008

page 228
Le syndrome de Marfan (SM) est une maladie génétique due à une altération de la synthèse de la fibrilline type I qui représente une des composantes du tissu conjonctif. Ce syndrome peut atteindre plusieurs organes

Sa fréquence est d'environ de 2-3 cas/10.000 [1]. Les symptômes apparaissent progressivement dès l'enfance, notamment l'allongement osseux, l'ectopie du cristallin et surtout l'anévrisme de la racine de l'aorte qui, avec son risque de dissection, conditionne le pronostic vital ; ces trois éléments constituent la triade caractéristique [2]. Le pronostic s'est beaucoup amélioré avec le traitement chirurgical précoce de la dilatation aortique et maintenant l'espérance de vie est presque identique à celle de la population générale alors qu'elle était autrefois inférieure à 40 ans.

La prise en charge des patients est pluridisciplinaire, avec un traitement prophylactique systématique pour l'atteinte de l'aorte afin d'intervenir avant la survenue de complications sérieuses [3].

\section{CAS CLINIQUE}

M. Omar M., âgé de 17 ans, étudiant, vient consulter, accompagné de sa sœur, au Centre de consultation et de traitements dentaires. II souhaite le remplacement de la 11 par implant et il a déjà effectué plusieurs consultations et devis chez des praticiens privés.

Le patient est atteint d'un SM diagnostiqué à l'âge de 10 ans (2001), et dès lors une surveillance médicale a été instaurée II n'y a aucune notion d'alcoolotabagisme. Dans la fratrie (2 frères et 2 sœurs), 3 (2 frères et 1 sœur) sont de grande taille mais ils ne sont pas atteints d'un SM (Fig. 1).

A l'âge de 11 ans, le patient a été mis sous bétabloquant $\left(\right.$ Avlocan $^{\circledR}$ ) car il avait un rythme cardiaque trop élevé. A l'âge de 13 ans (2003), une prothèse articulaire de la hanche et une plaque d'ostéosynthèse de la jambe ont été posées (accident en jouant au basket-ball).

A l'examen exo-buccal, il n'y a pas d'asymétrie faciale (Fig. 2) et on observe un endognathisme mandibulaire, et une augmentation de la taille de l'étage inférieur (Fig. 3).

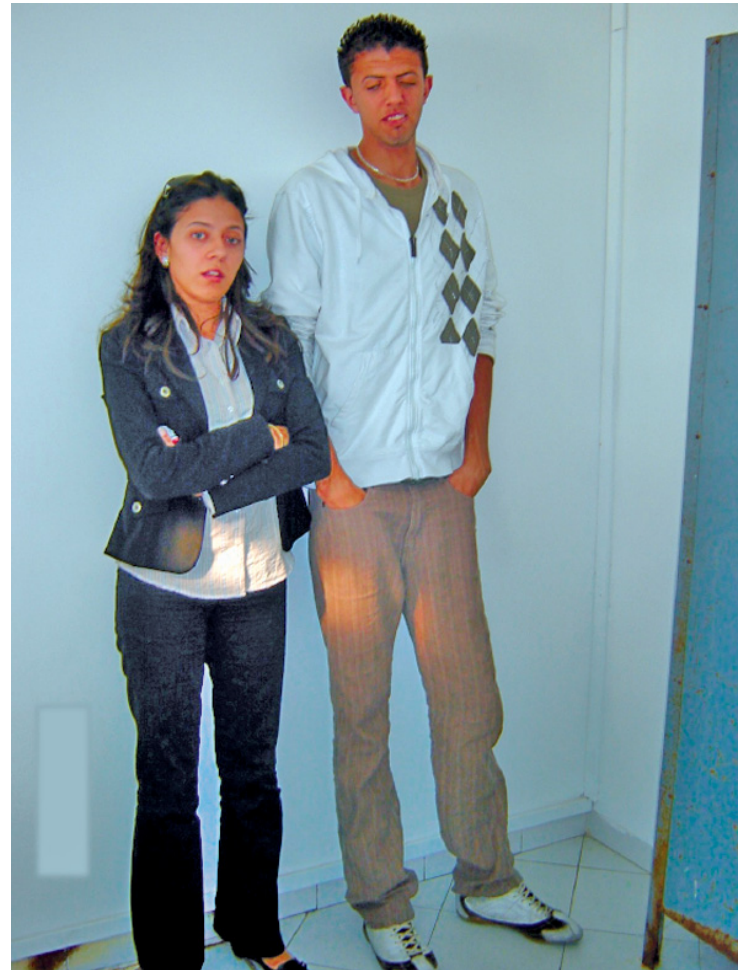

Figure 1: Gigantisme avec une taille $>190 \mathrm{~cm}$. Sœur de taille normale.

Gigantism with a size $>190 \mathrm{~cm}$. Sister of normal size.

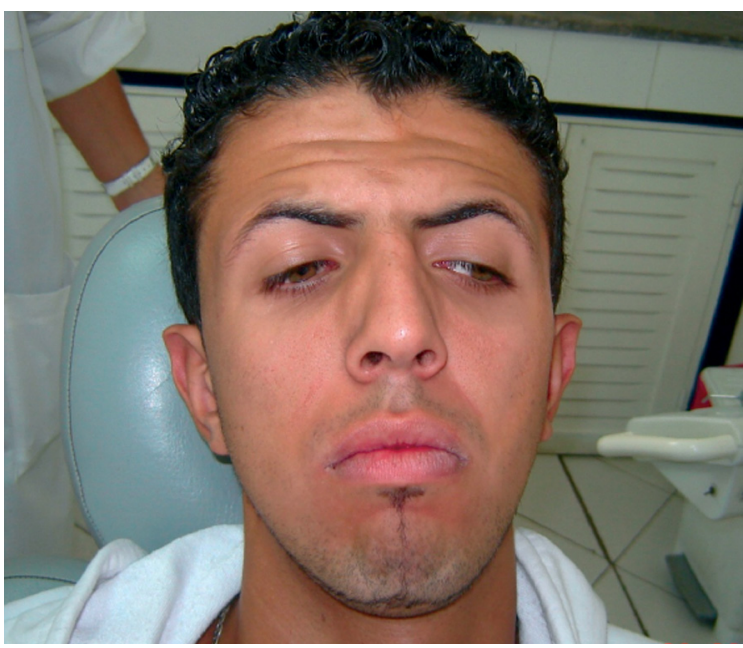

Figure 2 : Symétrie faciale respectée. Facial symmetry.

A l'examen endo-buccal, on note une mauvaise hygiène bucco-dentaire (Fig. 4) et surtout une absence de motivation pour l'améliorer.

Le patient a déjà eu des soins conservateurs et, chaque fois, les suites du traitement ont été marquées par un épisode inflammatoire et l'apparition 


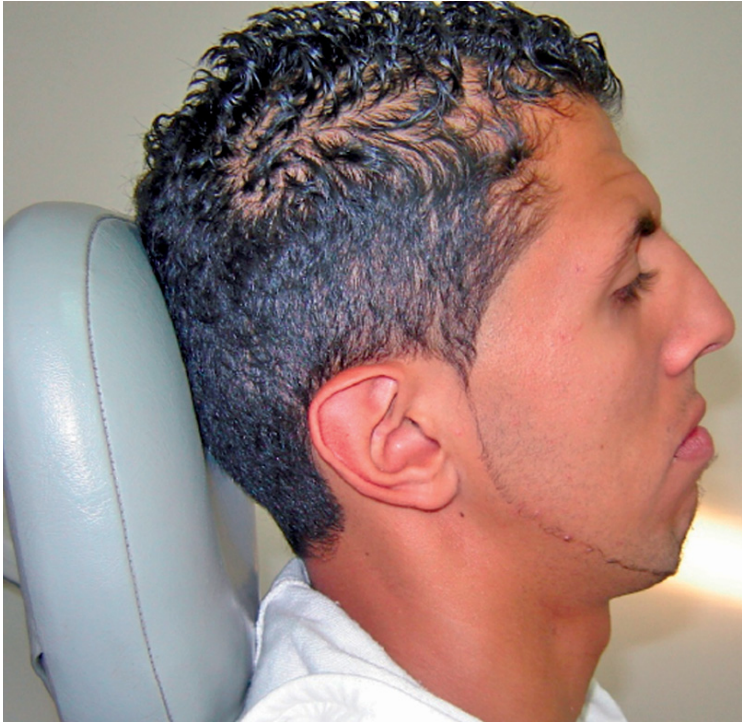

Figure 3 : Profil convexe et endognathie mandibulaire. Convexe side and mandibular endognathie

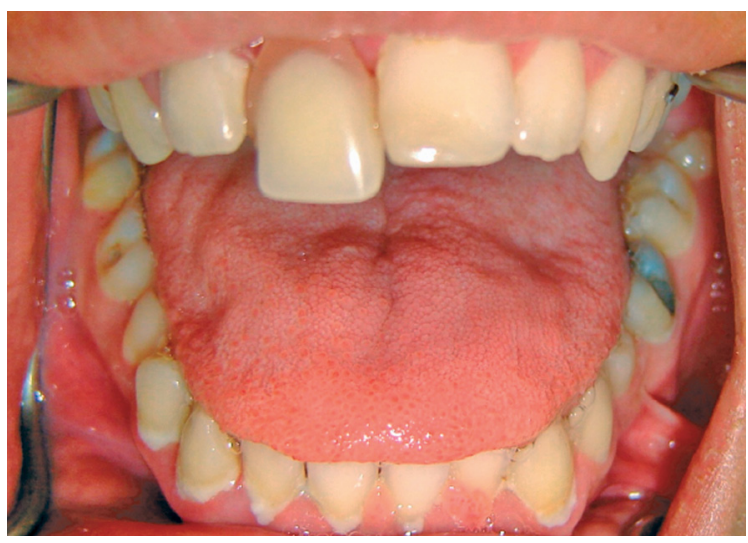

Figure 4 : Mauvaise hygiène bucco-dentaire et obturations coronaires défectueuses.

Poor oral hygiene and defective coronal fillings.

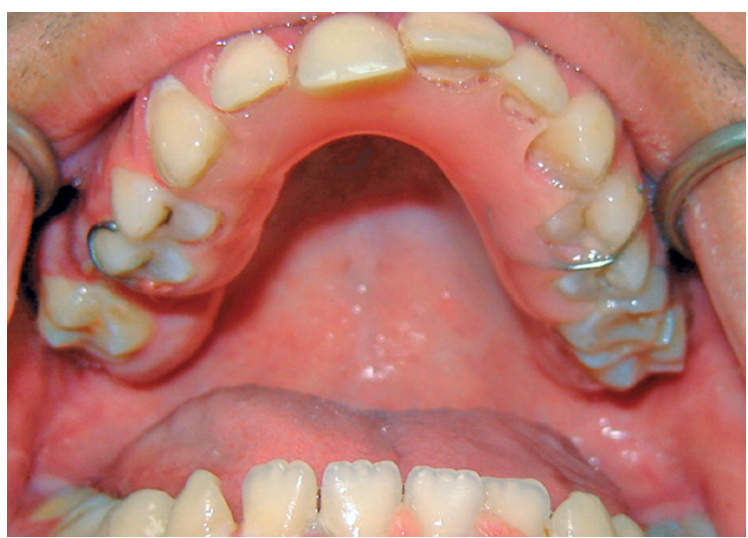

Figure 5 : Prothèse provisoire maxillaire mal adaptée. Inappropriate provisional maxillary prosthesis. d'« aphtes ». Un traitement orthodontique a été entrepris mais il a du être arrêté après 6 mois de traitement en raison des complications locales (inflammation, " aphtes »).

La perte de la 11 est secondaire à une carie délabrante et une inflammation gingivale ; elle a été remplacée par une prothèse amovible provisoire depuis 3 ans (Fig. 5).

Sur le plan occlusal, le patient présente une endoalvéolie bi-maxillaire et une béance antérieure (Fig. 6).

Au terme de l'examen, on peut résumer le dossier médical ainsi :

- SM et prothèse de la hanche,

- Gingivite due à la plaque,

- Nécrose de la 16, des caries sur 25, 27, 37, 46, 17,16 et 15 , et obturations défectueuses sur 21 , 26, 36 et 46 ,

- Edentement antérieur (la 11), endo-alvéolie bimaxillaire et béance antérieure.

Au total, au terme de ce bilan, peut-on répondre favorablement à la demande du patient et de sa famille et réaliser une chirurgie implantaire pour le remplacement de la 11 ? L'état général de ce patient le permet-il ?

\section{DISCUSSION}

Les différents facteurs qui interviennent dans la décision thérapeutique sont :

- Les facteurs généraux induits par le SM,

- Les facteurs locaux liés au patient,

- Les facteurs locaux en rapport avec les facteurs généraux.

\section{Les facteurs généraux induits par le SM}

Le SM est une maladie génétique du tissu conjonctif, de transmission autosomique dominante, généralement en rapport avec une anomalie du gène de la fibrilline, qui se traduit par l'atteinte de nombreux systèmes, plus particulièrement les systèmes cardio-vasculaire, oculaire, et musculo-squelettique. Les manifestations cardiovasculaires sont surtout dues à la fragilité de la racine de l'aorte avec risque de dilatation puis de dissection ; ceci représente le principal facteur de morbidité et de mortalité de la maladie. médecine

buccale

chirurgie

buccale

VOL. $14, N^{\circ} 4$

2008

page 229 
médecine

buccale

chirurgie

buccale

VOL. $14, \mathrm{~N}^{\circ} 4$ 2008

page 230
II peut y avoir d'autres manifestations cardiovasculaires : prolapsus mitral, dilatation ventriculaire, défaillance cardiaque ${ }^{[1,4]}$.

Le patient souffre d'une arythmie cardiaque et a été mis sous béta-bloquant (Avlocan ${ }^{\circledR}$ ). En l'absence d'autres manifestations cardiovasculaires, ce patient ne présente pas de risque d'endocardite bactérienne mais, avec l'évolution du SM, un risque d'endocardite bactérienne risque d'apparaitre tôt ou tard. Selon l'agence française de sécurité sanitaire des produits de sante (AFSSAPS), en raison de la présence d'une prothèse de hanche, ce patient est classé dans la catégorie $B$ des patients à risque chez qui la chirurgie implantaire constitue une contre-indication absolue.

Par ailleurs, dans le SM, il existe une ostéoporose[5] et une ostéopénie [6]. Toutefois plusieurs études ont montré qu'il n'y a pas de différence significative entre l'ostéointégration des implants chez les patients sains et celle chez les patients ayant une ostéoporose. Ce résultat parodontal tient sans doute au fait que l'ostéoporose touche surtout les os longs ${ }^{[7]}$. En conséquence, l'oséointégration d'un l'implant dentaire doit s'effectuer normalement chez un patient atteint d'un SM.

\section{Les facteurs locaux liés au patient}

Le patient présente un mauvais état d'hygiène bucco-dentaire (Fig. 4), ce qui représente un risque pour la pérennité de l'implant dentaire. Malgré les nombreux conseils de brossage et d'hygiène, le patient n'a jamais semblé être réellement motivé. Cette absence de motivation constitue une contre-indication relative à la mise en place d'un implant ${ }^{[7,8]}$.

\section{Les facteurs locaux induits par le SM}

Des études in vitro et in vivo ont montré la présence d'altération structurale des tissus parodontaux lors qu'il y a une altération de la fibrilline type I. Ces altérations, aggravées par la plaque et la mauvaise hygiène bucco-dentaire, aboutissent à des parodontites agressive ou chronique $[9,10,11]$. Ceci constitue une contre-indication relative à la pose d'implant [7].

\section{DECISION THERAPEUTIQUE}

Pour ce patient il existe une contre-indication absolue et plusieurs contre-indications relatives à la pose d'un implant dentaire (Tab. 1). En conséquence il a été déconseillé au patient d'envisager ce traitement et il a été orienté vers une prise en charge orthodontique - parodontale et une réhabilitation prothétique fixe conventionnelle.

Figure 5 : Tableau synoptique des contre-indications à la pose d'un implant dentaire chez ce jeune patient atteint d'un SM.

Synopsis of contraindications against the installation of a dental implant in young patients with MS.

\begin{tabular}{|c|c|c|}
\hline & $\begin{array}{c}\text { Contre-indications } \\
\text { relatives }\end{array}$ & $\begin{array}{c}\text { Contre-indications } \\
\text { absolues }\end{array}$ \\
\hline $\begin{array}{l}\text { Facteurs généraux : (Syndrome de Marfan) } \\
\text { - Complications cardio-vasculaires } \\
\text { - Ostéoporose, ostéopénie }{ }^{[7]} \\
\text { - Prothèse articulaire (patient à risque B, AFSSAPS } \\
\text { 2001) }\end{array}$ & $\begin{array}{l}- \\
- \\
-\end{array}$ & $\begin{array}{c}-(+) \\
- \\
+\end{array}$ \\
\hline $\begin{array}{l}\text { Facteurs locaux } \\
\text { - Mauvaise hygiène bucco-dentaire } \\
\text { - Absence de motivation }\end{array}$ & $\begin{array}{l}+ \\
+\end{array}$ & $\begin{array}{l}- \\
-\end{array}$ \\
\hline $\begin{array}{l}\text { Facteurs locaux induits par le SM } \\
\text { - Altération des tissus parodontaux } \\
\text { - Résorption osseuse marginale }\end{array}$ & $\begin{array}{l}+ \\
+\end{array}$ & - \\
\hline
\end{tabular}




\section{RÉFÉRENCES}

1 - Beylot C, Martin L. Maladies héréditaires du collagène et du tissu élastique. Encycl Med Chir 98-770-A-10, 2007.

2 - Edouart T, Tauber M. Avance staturale et gigantisme. Encycl Med Chir 10-033-E-10, 2008.

3 - lung Bb, Iserin L. Cardiopathies et grossese. Encycl Med Chir 5-044-A-10, 2007.

4 - John CS D. Marfan syndrome: clinical diagnosis and management. Eur J Hum Genet 2007 ; 15: 724-733.

5 - Legrand E, Degasne I, Chappard D, Basle MF, Audran M. Ostéoporoses. Encycl Med Chir 14-027-G10, 1999.

6 - Moura B, Tubach F, Sulpice M, Boileau C, Jondeau G, Muti C, Chevallier B, Ounnoughene Y, Le Parc JM, Densité minérale osseuse et syndrome de Marfan. Rev Rhumat 2006 ; 73 : 1405-1408.
7 - Bartolucci E. Réussir les implants dentaires. Masson, Paris, 2006, 13-21.

8 - Dubruille JH, Dubruille MT, Goudot P, Muster D, Pigot JL, Vanhakendover S. Réhabilitation orale et implantologue. Encycl Med Chir 23-330-A-10, 2000.

9 - Grollmus N. Chávez M, Donat F. Periodontal disease associated to systemic genetic disorders. Med Oral Patol Oral Cir Bucal 2007 ; 12 : E211-5.

10 - Shiga M, Saito M, Hattori M, Torii C, Kosaki K, Kiyono T, Suda N. Characteristic phenotype of immortalized periodontal cells isolated from Marfan syndrome type I. Cell Tissue Res 2008, 331: 461-472.

11 - Von Kodolitsch Y, Robinson P. Marfan syndrome: an update of genetic medical and surgical managemnt. Heart 2007 ; $93:$ 755-60. \begin{tabular}{l}
$\begin{array}{l}\text { médecine } \\
\text { buccale } \\
\text { chirurgie } \\
\text { buccale }\end{array}$ \\
\hline VOL. $14, N^{\circ} 4$ \\
2008
\end{tabular}

page 231 